\title{
Alveolar ridge atrophy related to facial morphology in edentulous patients
}

\author{
This article was published in the following Dove Press journal: \\ Clinical Interventions in Aging \\ 13 September 2017 \\ Number of times this article has been viewed
}

\author{
Joanna Kućl \\ Teresa Sierpińska² \\ Maria Gołębiewska' \\ 'Department of Prosthodontics, \\ 'Department of Dental Technology, \\ Medical University of Bialystok, \\ Bialystok, Poland
}

Correspondence: Joanna Kuć Department of Prosthodontics, Medical University of Bialystok, M SklodowskaCurie Str. 24a, I5-276 Bialystok, Poland Tel +48 857468349

Fax +48 857447030

Email kucjoanna@poczta.onet.pl
Objectives: The morphology of the alveolar process determines the retention and stability of prosthetic restorations, thereby determining the result of the therapy. Considering that the edentulous jaws may be affected by the atrophy process, it was hypothesized that the morphology of the alveolar process of the maxilla may be dependent on the anterior facial height and anatomy of the mandible.

Subjects and methods: Twenty-five healthy edentulous Caucasian individuals were randomly chosen. Each subject underwent a lateral cephalogram before and after prosthetic rehabilitation. During exposition, newly made prostheses were placed in the patient's mouth. Teeth remained in maximal intercuspidation. Morphological parameters were evaluated according to the Ricketts, McNamara, and Tallgren's method.

Results: An inversely proportional association was observed between patient age and the distal part of the maxilla. A statistically significant connection was noted between the vertical dimension of alveolar ridge and anterior total and lower facial height conditioned by prosthetic rehabilitation.

Conclusion: The height of the lateral part of the alveolar ridge of the maxilla remains in connection with the anterior total and lower facial height obtained in the course of prosthetic rehabilitation. The vertical dimension of the alveolar ridge of the maxilla seems to be in close relationship with the morphology of the lower jaw.

Keywords: anterior facial height, cephalometric analysis, complete dentures, vertical occlusal dimension

\section{Introduction}

Alveolar bone morphology plays a significant role in the rehabilitation of the edentulous patient. It determines the retention and stability of prosthetic restorations, thus determining the result of the therapy. While the influence of the shape and dimensions of the prosthetic base is well known, the role of the vertical relationship of facial morphology is often underestimated.

A study of morphological changes in the facial skeleton of complete denture wearers reveals that mean reduction in anterior height of the maxillary process is four times smaller than that of the alveolar part of the mandible. ${ }^{1}$ The reasons are a wider surface area and thin cortical and thick trabecular bone structure of the maxilla that allow for easier transference and distribution of biting forces. ${ }^{2}$ Resorption of the residual alveolar ridges results in forward-upward rotation of the mandible, decreases in the occlusal vertical dimension, and increases in the mandibular prognathism (the class III tendency). ${ }^{3-6}$ Sometimes, it is possible to state a reduction in the horizontal overlap or even negative overjet where the upper incisors are behind 
the lower ones. ${ }^{2}$ It should be mentioned that in special cases of treatment with complete dentures, occlusal vertical dimension is often intentionally reduced in order to achieve denture stability. ${ }^{7}$

The knowledge of angulation and direction of the bony residual ridge to the horizontal plane facilitates prosthetic rehabilitation. It indicates positioning of implants or placement of acrylic teeth in removable dentures. ${ }^{8}$ Morphological changes of edentulous jaws follow a predictable pattern after tooth extraction. ${ }^{8}$ This was emphasized by Cawood and Howell in their classification. ${ }^{9}$

Alveolar bone morphology is multi-dimensionally conditioned. The presence of natural teeth influences a positive effect on mature jaws. ${ }^{10}$ The alveolar bone structure is formed by the following local and anatomical factors: mechanical compression, ${ }^{11,12}$ use or nonuse of dentures, denture-wearing time, edentulous period, number of sets of prosthetic restorations, ${ }^{12}$ muscle function and activity, ${ }^{11}$ skeletal pattern, ${ }^{1}$ and bone quality. ${ }^{11,13}$ A special role is ascribed to age, ${ }^{12}$ gender, ${ }^{11,14}$ hormones, ${ }^{11}$ metabolic diseases, ${ }^{11,15}$ and osteoporosis. ${ }^{11}$

The essence of rehabilitation of the edentulous is to restore the proper vertical and horizontal relationship within the facial part of the skull, with the correct position of the mandibular condylar head within articular fossa, compliance of the body and mandibular symphysis course, as well as the maximum intercuspation of both dental arches and facial harmony. The difficulty in the treatment of complete denture wearers results from innumerable possible positions of the edentulous mandible that may be taken in respect to the maxillary base.

Our study objective was to assess a potential link between anterior facial height and alveolar process of the maxilla in edentulous patients. Bearing in mind that retention and stability of conventional dentures are closely related to alveolar bone morphology, it was hypothesized that there exists potential link between age, denture-wearing time, edentulous period, and morphological parameters of the edentulous ridge of the maxilla. It was expected that there are some relationships between alveolar bone morphology and vertical occlusal dimension (anterior facial height). It was predicted that alveolar bone morphology is strongly associated with alveolar part of the mandible.

\section{Subjects and methods Subjects and sample size}

A total of 25 healthy Caucasian people were randomly chosen, 14 women and 11 men (age 44-86 years; mean $=70.5 \pm 9$ ), with a total deficiency of teeth. All study participants underwent prosthetic treatment, including fabrication of new complete dentures.

Inclusion criteria were the use of prosthetic appliances (complete dentures) showing signs of artificial tooth wear and the clinical unhealthy condition of central occlusion before therapy [flat surface of the artificial teeth, lack of intercuspidation, (pseudo) mandibular prognathism, and occlusal vertical dimension disturbances - too high or too low] and good conditions after treatment. The mean denture-wearing time was 9.8 \pm 5.2 years, and mean duration of complete lack of teeth was $17 \pm 10$ years. Twentythree patients replaced their dentures twice during the entire period of being edentulous. Patients suffering from severe chronic diseases were not engaged to participate in the research. People affected by disorders of hard and soft tissues of the mouth, jaw dysfunction, and abnormalities that affected jaw position were also excluded. None of the members in this study group declared a history of orthodontic treatment.

\section{Conditions which required patient exclusion}

Respiratory diseases including asthma, chronic obstructive pulmonary disease, and emphysema; Metabolic disorders including diabetes and calcium and phosphate dysregulation; Corticosteroid therapy; Alcohol dependence; Ellisworth Kelly's syndrome; Notable craniofacial asymmetry, including hemifacial microsomia, hemimandibular hypoplasia, and acromegaly; Hormone replacement therapy; Tuberosity down growth (with or without maxillary sinus pneumatization); Flabby ridges and soft tissue hyperplasia; Burning mouth syndrome and orofacial complaints including neuralgias; Injuries within craniocervical, craniomandibular, or craniofacial areas requiring surgical intervention; Diseases associated with low bone mass.

\section{Conventional complete dentures fabrication}

All patients received new conventional complete upper and lower dentures. Current standards of treatment were followed. For the initial impression, alginate was used (Hydrogum, Zhermack). The final impression was performed by using a custom acrylic resin tray with molded border and C-silicone (Xantopren M mucosa, Heraeus Kulzer; Oranwash L, Zhermack). The conventional method for registration of the central occlusion was undertaken. To establish maxillomandibular relationships, wax occlusion 
rims were used. The maxillary wax rim was reduced to approximately 1-2 $\mathrm{mm}$ below the lower edge of the lip. The posterior part of the upper rim in the first molar area was formed such that the edge of the rim was approximately 0.25 -inch below Stensen's duct. The occlusal plane was modified until it was parallel to the Camper's and interpupillary lines. Camper's line was defined as a line projected from the ala of the nose to the superior edge of the tragus of the ear. The lower rim was trimmed and countered until it met the upper rim, approximately $3 \mathrm{~mm}$ less than the measurement for the vertical dimension at rest. The resting position of the mandible was physiologically determined. Patients were asked to undertake the following actions: swallow, say " $\mathrm{m}$ " and then relax. In order to minimize errors associated with the determination of centric occlusion, these steps were repeated several times. The distance between the reference points (Sn' - subnasale, Me' - soft tissue menton) was recorded. Willis' device for facial measurements was used. To establish horizontal jaw relationship, the clinician's index finger was placed on the buccal surface of the mandibular rim in both premolar areas, with the thumbs positioned within the patient's chin. The patient was informed to close slowly and gently on the back teeth. The centric occlusion record was transferred to the semi-adjustable articulator. During the clinical procedure, facebow were also applied. Anatomically shaped acrylic teeth with $33^{\circ}$ cuspal inclination were used.

\section{Radiological procedure}

Each participant underwent a lateral radiograph. The projections were made in the standing posture. The head was orientated with respect to the cephalostat. During exposition, newly made prostheses were placed in the patient's mouth. Teeth remained in maximal intercuspidation. Patients were asked not to swallow during exposures. All cephalograms were taken by a single technician. The same $\mathrm{X}$-ray device was used in all cases. The generally accepted procedure of lateral cephalograms was applied: the distance between the focus and the mid-sagittal plane was $152 \mathrm{~cm}$ and that between the film and the mid-sagittal plane was $10 \mathrm{~cm}$. The final enlargement of the actual dimension was $8 \%-14 \%$. The results were not corrected due to the linear increase.

Each radiograph required an X-ray dose of $0.15 \mathrm{mSv}$. The mean exposure values were as follows: $77 \mathrm{kV}, 12 \mathrm{~mA}$, for $0.64 \mathrm{~s}$. Standard-size radiographic films $(18 \times 24 \mathrm{~cm})$ with a resolution of $0.35-0.5$ were used. Cephalometric landmarks were transferred on tracing paper $(210 \times 297 \mathrm{~mm}$ A 4, $\left.90 \mathrm{~g} / \mathrm{m}^{2}-\mathrm{gsm}\right)$. All radiological measurements were made manually by one researcher. The initial position of the reference points was re-evaluated by two examiners due to the possible appearance of drawing errors.

\section{Cephalometric study}

Radiological measurements were conducted with respect to:

1) Ricketts ${ }^{16-19}$ and McNamara ${ }^{19,20}$ cephalometric analysis (Figure 1).

2) Tallgren's method ${ }^{21}$ (Figure 2).

The following parameters related to Ricketts and McNamara were noted:

A. indicators of vertical component of the centric occlusion:

a. within soft tissue:

- anterior total facial height described as G'Me'

- anterior lower facial height reported as Sn'Me'

b. within hard tissue:

- anterior total facial height noted as nasomental distance $(\mathrm{NMe})$

- anterior lower facial height considered as AnsMe

- angular measurement of the anterior lower facial height with respect to the center of the ramus mandible noted as AnsXiPm

B. measurements that describe the relationship between maxilla and the cranial base:

a. within soft tissue:

- G'Sn' - anterior upper facial height

b. within hard tissue:

- SNA - indicates anteroposterior position of the maxilla with respect to the cranial base (normal position, retrognathic or prognathic)

- NFcA - angular measurement between the nasion, facial center, and subspinale

- NAns - anterior upper facial height

C. other cephalometric measurements:

- facial angle - angular measurement between the $\mathrm{BaN}$ and PTGn lines; in people with natural dentition indicates vertical or horizontal facial growth, defines tendency to skeletal class II or III

- $\mathrm{SNB}$ - indicates position of the mandible with respect to the cranial base

- ANB - determines mutual position of the jaws and, thus, skeletal classes

- $\mathrm{CoGn}$ - specifies mandibular length

- CoGo - defines height of the ramus of the mandible

- mandibular angle 


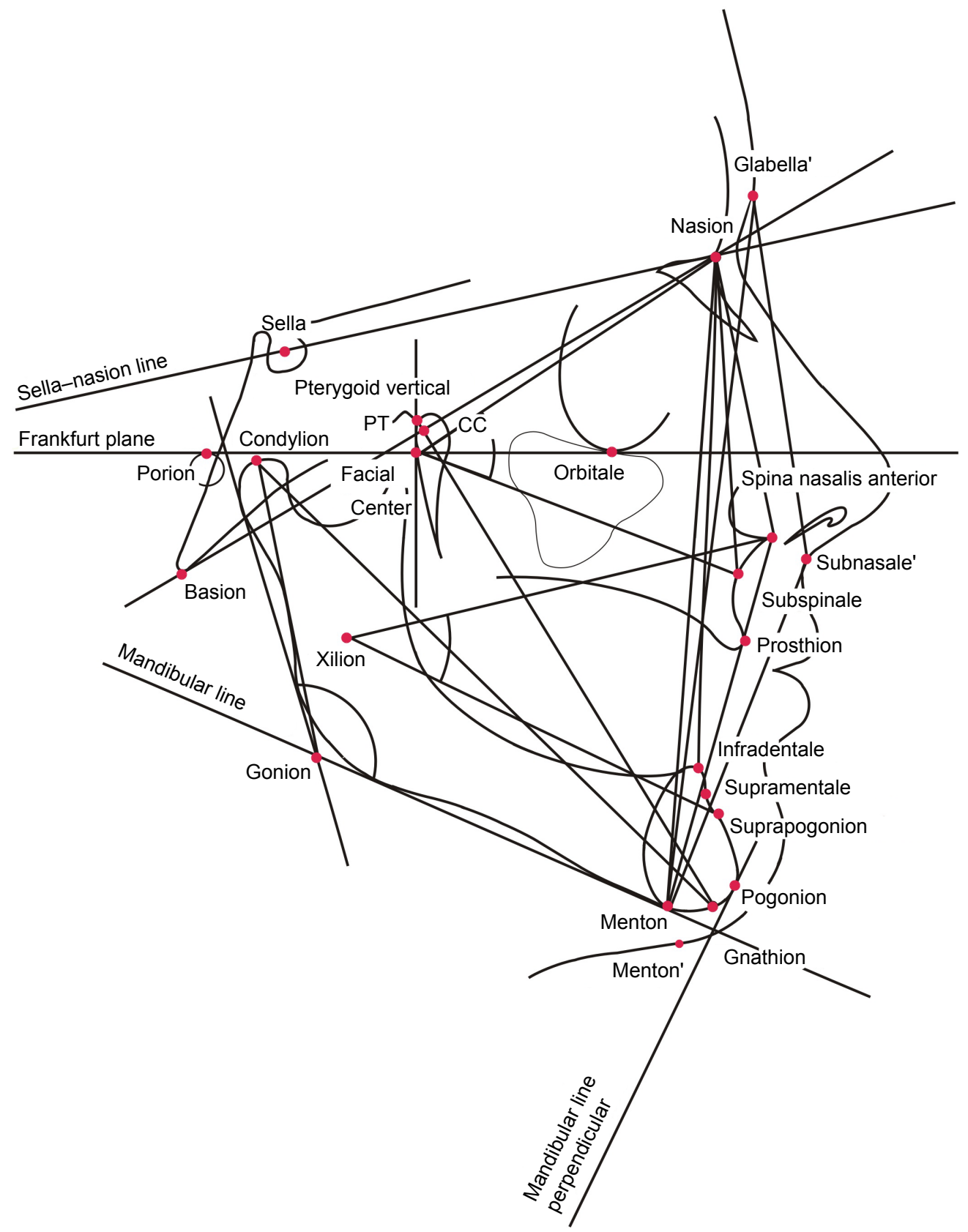

Figure I Reference points and lines with regard to the cephalometric analysis. A detailed description of the reference points is presented in Table I.

Notes: ' indicates that the points are located within soft tissue. Kuć J, Sierpińska T, Gołębiewska M. The relationship between facial morphology and the structure of the alveolar part of the mandible in edentulous complete denture wearers. A preliminary study. Acta Odontologica Scandinavica. 20I 5;73(I):57-66. Copyright (C) Acta Odontologica Scandinavica Society, adapted by permission of Taylor \& Francis Ltd, www.tandfonline.com on behalf of Acta Odontologica Scandinavica Society. ${ }^{19}$

Abbreviations: CC, center of the cranium; PT, point at the junction of the pterygopalatine fossae and foramen rotundum placed at II o'clock from the pterygomaxillary fissure.

Table 1 contains landmarks considered with regard to Ricketts and McNamara measurements.

The following landmarks were considered with respect to Tallgren's method ${ }^{21}$ (Figure 2):

A. Points located within maxilla:

- $\mathrm{mx} 1$ - point which was situated as the most inferoposterior point on the tuberosity

- $\mathrm{mx} 5$ - point resulting from intersection between the horizontal and palatal ridge contours.
- $\mathrm{mx} 1, \mathrm{mx} 2, \mathrm{mx} 3, \mathrm{mx} 4$, and $\mathrm{mx} 5$ - points within the contour of the alveolar process located at equal distances from each other with respect to the AnsPns line

- $\mathrm{mx} 6$ - point situated within the top of the anterior part of the alveolar ridge in relation to the AnsPns line

- $m \times 7$ - defined as a prosthion: the most inferior point on the anterior contour of the maxillary alveolar process 


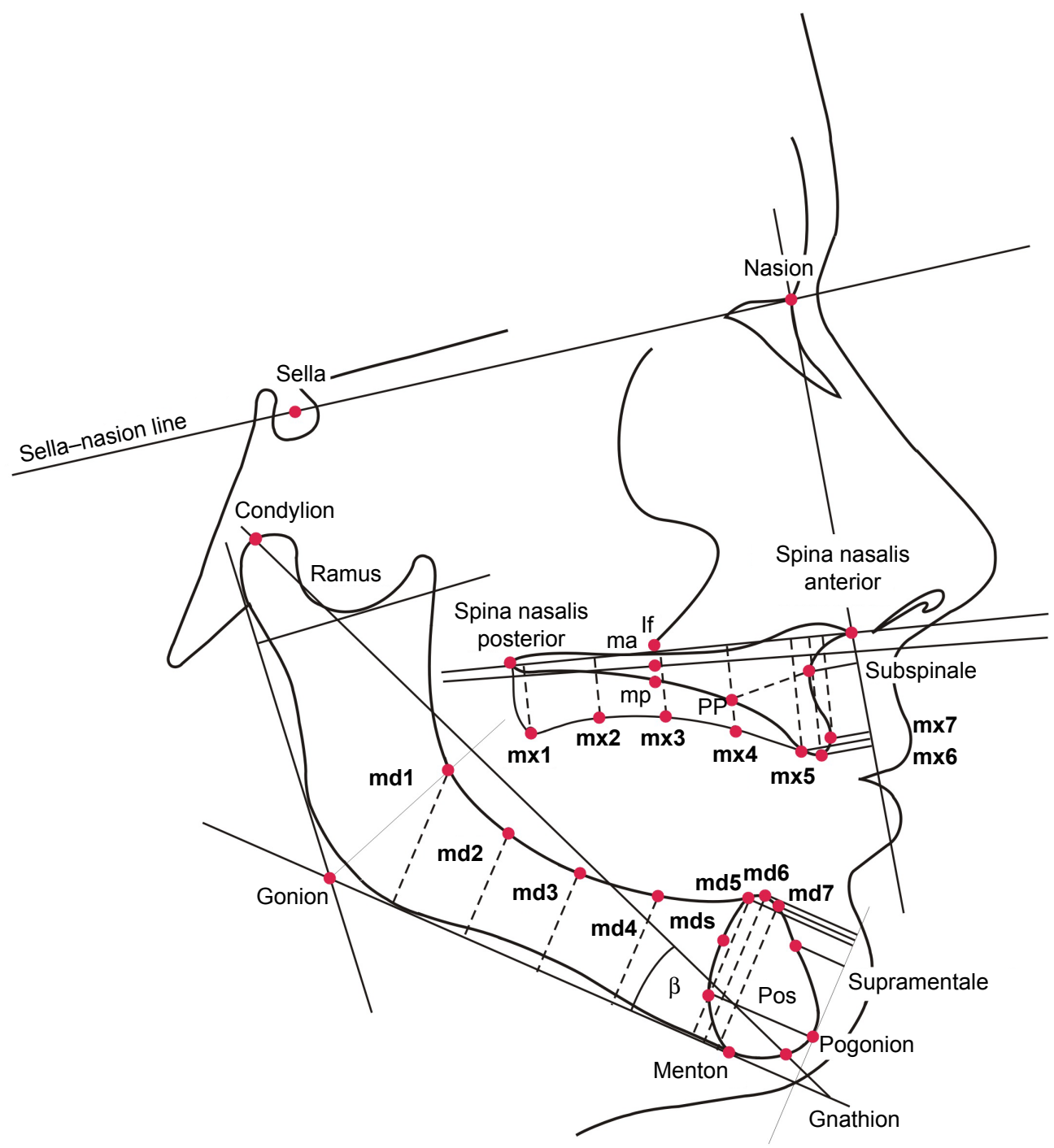

Figure 2 Reference points with regard to the analysis of the morphology of the alveolar part of the mandible. A detailed description of the reference points is presented in Table I and in the second paragraph of the study - Subjects and methods.

Notes: $\mathrm{mdl}, \mathrm{md} 2, \mathrm{md} 3, \mathrm{md} 4, \mathrm{md} 5$ - points located equidistant from each other with respect to the mandibular plane; md5 - point situated within the mandible center line, noted in the most posterior and upward direction; md6 - the highest point situated within the mandible center line with regard to the mandibular plane; md7 noted as the point within the mandible center line, situated in the most anterior and upward direction. $\mathrm{m} \times \mathrm{I}, \mathrm{m} \times 2, \mathrm{~m} \times 3, \mathrm{~m} \times 4, \mathrm{~m} \times 5-$ points within the contour of the alveolar process located at equal distances from each other with respect to the AnsPns line; mx6 - point situated within the top of the anterior part of the alveolar ridge in relation to the AnsPns line; $\mathrm{m} \times 7$ - the most inferior point on the anterior contour of the maxillary process. Pos, localized as the intersection between the posterior contour of mandibular symphysis and a line through Pg parallel to ML. PP, the point which is localized midway between points $\mathrm{mp}$ and $\mathrm{mx5}$. If, inframalar point. mp, projection of inframalar point on the inferior contour of hard palate. ma, maxillon. the midpoint of the hard palate. Kuć J, Sierpińska T, Gołębiewska M. The relationship between facial morphology and the structure of the alveolar part of the mandible in edentulous complete denture wearers. A preliminary study. Acta Odontologica Scandinavica. 2015;73(I):57-66. Copyright @ Acta Odontologica Scandinavica Society, adapted by permission of Taylor \& Francis Ltd, www.tandfonline.com on behalf of Acta Odontologica Scandinavica Society. ${ }^{19}$

- $\mathrm{SS}(\mathrm{A})$ - point located between the anterior nasal spine (Ans) and prosthion $(\mathrm{mx} 7)$ as the lowest point of the curve

- $\mathrm{PP}$ - the point which was localized midway between the mp point and $\mathrm{mx} 5$

- $\mathrm{mp}$ - defined as the projection of the inframalar point on the inferior contour of hard palate

- If - inframalar point: localized on the inferior-most contour of the malar bone

B. Points located within mandible:
- $\operatorname{md} 1$ - noted as the point located in the place where the bisector of the mandibular angle intersects the retromolar triangle

- md5 - point situated within the mandible center line, noted in the most posterior and upward direction

- md1, md2, md3, md4, and md5 - points located equidistant from each other with respect to the mandibular plane

- md6 - the highest point situated within the mandible center line with regard to the mandibular plane 
Table I Reference points and lines

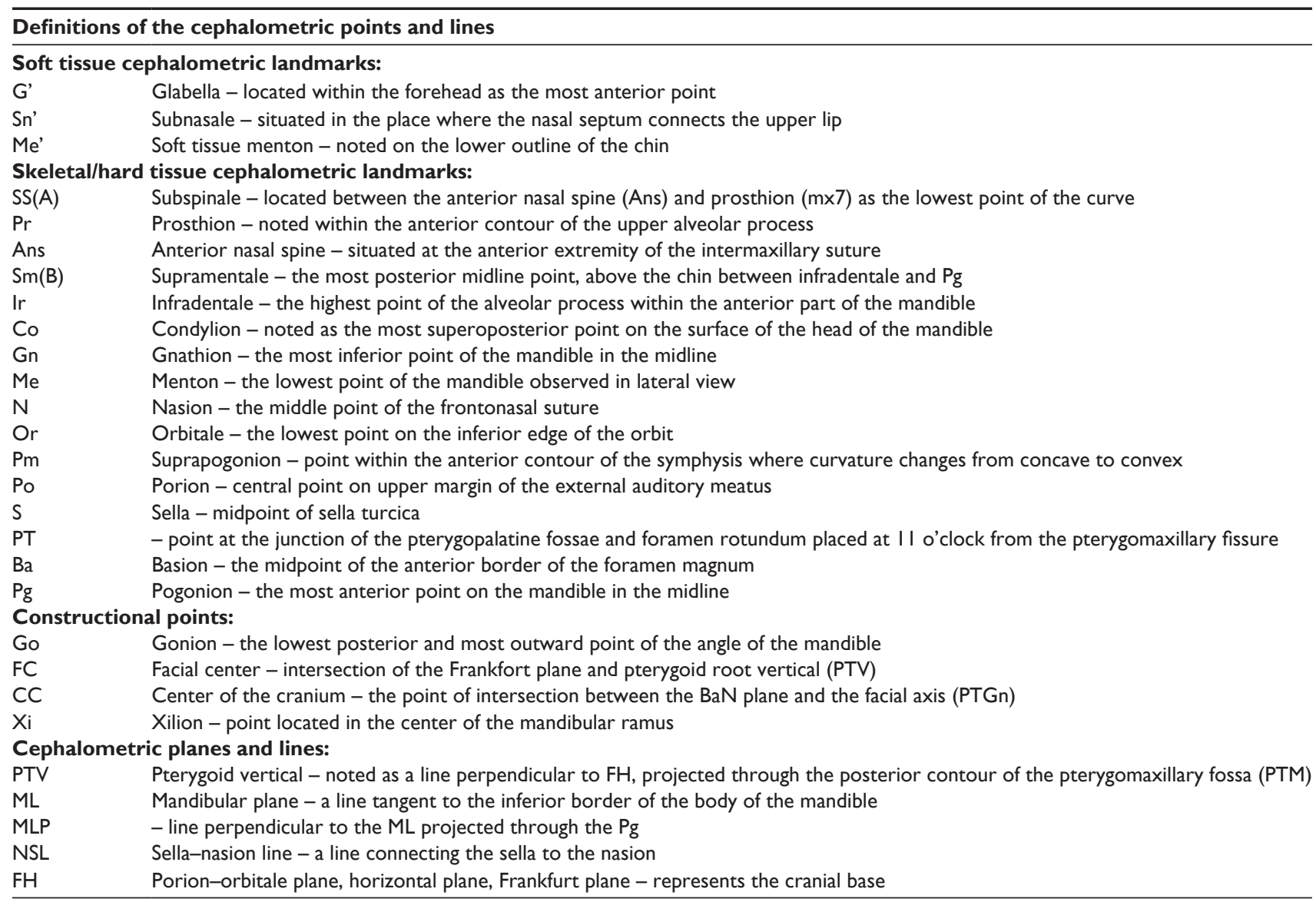

Note: Kuć J, Sierpińska T, Gołębiewska M. The relationship between facial morphology and the structure of the alveolar part of the mandible in edentulous complete denture wearers. A preliminary study. Acta Odontologica Scandinavica. 2015;73(1):57-66. Copyright @ Acta Odontologica Scandinavica Society, adapted by permission of Taylor \& Francis Ltd, www.tandfonline.com on behalf of Acta Odontologica Scandinavica Society. ${ }^{19}$

- $\operatorname{md} 7$ - noted as the point within the mandible center line, situated in the most anterior and upward direction

- $\mathrm{Sm}(\mathrm{B})$ (supramentale) - located between infradentale (Ir) and pogonion $(\mathrm{Pg})$ in the deepest place of the curve

- Pos - localized as the intersection between the posterior contour of the mandibular symphysis and a line through Pg parallel to $\mathrm{ML}$

- mds - situated on the posterior contour of the mandibular symphysis as the mid-point between point Pos and md5

C. Additional cephalometric measurements:

- $\beta$ - considered as an angle between the mandibular plane ML and mandibular length (CoGn)

- $\mathrm{Ra}$ - the width of the ramus of the mandible specified by the distance between anatomic borders of the ramus ascendens of the mandible

- anteroposterior dimension of the symphysis:

- $\operatorname{mds}-\mathrm{Sm}(\mathrm{B})$

- $\mathrm{Pos}-\mathrm{Pg}$

\section{General description of the method}

The study included the following:

1) assessment of the quality of clinical results of prosthetic rehabilitation in respect to reference value of the AnsXiPm angle based on comparison of the first and second radiograph received before and after the treatment, respectively - considered as an inclusion criteria

2) evaluation of the maxilla morphology:

- assessment of the distances between the position of the reference points $\mathrm{mx} 1 \rightarrow \mathrm{mx} 7, \mathrm{SS}(\mathrm{A})$, and the AnsPns line,

- assessment of the distances between position of the reference points $\mathrm{mx} 5 \rightarrow \mathrm{mx} 7, \mathrm{SS}(\mathrm{A})$, and the SNAns line;

- evaluation of the cross section of the anterior part of the alveolar process (PP-A)

3) assessment of the potential link between the shape of the edentulous maxilla and age of the patients, denture-wearing period, and duration of edentulousness 
4) evaluation of the possible connection between measurements associated with anterior total, lower, and upper facial height and edentulous maxilla

5) observation of potential link between other measurements in respect to Ricketts and McNamara analysis and the edentulous maxilla

6) assessment of potential link between the edentulous maxilla and mandible

All radiological measurements from 2 to 5 were conducted with regard to the second radiograph (cephalogram performed after prosthetic rehabilitation on the first day of using new dentures). This article is a continuation of the earlier study on the relationship between the mandible and facial morphology in edentulous complete denture wearers. ${ }^{19}$

\section{Statistical analysis}

Statistical analysis was conducted using the Statistica 7.0 software. Measurements of the parameters were calculated three times, and the values received were averaged. The measure of central tendency (including arithmetic mean, median) and measures of variation (standard deviation) were used to assess the distribution of quantitative variables. The strength of the relationship between tested parameters was based on Spearman's rank correlation coefficient. Relationships were found to be statistically significant at $P<0.05$.

\section{Ethical approval}

The research protocol has gained the recognition of the Institutional Bioethic Committee at the Medical University of Bialystok, Poland (approval number: R-I-002/88/2008). All procedures were in accordance with the ICH Guideline for Good Clinical Practice. The protocol conformed to the criteria of the Declaration of Helsinki. Patients were enrolled into the study after obtaining their written informed consent.

\section{Results}

Table 2 provides mean value, standard deviation, and median of measurements of the maxilla with regard to Tallgren's method. ${ }^{21}$ The highest values of the appropriate parameters were observed in the anterior part of the alveolar process $\mathrm{mx} 4(\mathrm{Me}=16.00 \mathrm{~mm}), \mathrm{mx} 5(\mathrm{Me}=15.19 \mathrm{~mm})$, and $\mathrm{mx} 6$ $(\mathrm{Me}=15.88 \mathrm{~mm})-$ with respect to the AnsPns line. The lowest values were noted in the $\operatorname{Ss}(\mathrm{A})(\mathrm{Me}=5.75 \mathrm{~mm})$ and $\mathrm{mx} 1$ area $(\mathrm{Me}=10.56 \mathrm{~mm})$.

Table 3 presents relationships between the age of the patients, period of denture use, duration of edentulousness, and morphology of the maxilla. Inversely proportional connections with moderate degrees of severity were noted between age and measurements associated with the posterior part of the alveolar process $(\mathrm{mx} 1, \mathrm{mx} 2, \mathrm{mx} 3$, and $\mathrm{mx} 4$; $P<0.05)$. There was no correlation between skeletal measurements and denture-wearing time or period of edentulousness $(P>0.05)$.

Table 4 shows mutual implications of cephalometric linear indices (according to Ricketts and McNamara) with measurements of edentulous maxilla (with regard to Tallgren). Statistically significant values $(P<0.05)$ were observed between $\mathrm{mx} 1 \rightarrow \mathrm{mx} 6 /$ AnsPns and G'Me', Sn'Me', $\mathrm{NMe}$, AnsMe - measurements associated directly with anterior total and lower facial height. Mandibular measurements, described as $\mathrm{CoGn}$ and $\mathrm{CoGo}$, remained in a proportional relationship with respect to $\mathrm{mx} 2 \rightarrow \mathrm{mx} 4 /$ AnsPns (Table 4).

The lack of a connection was stated between skeletal pattern and the vertical dimension of the alveolar process (ANB angle; $P<0.05$; Table 5).

In terms of angular parameters, a relevant correlation was obtained for AnsXiPm/mx4/AnsPns and SNA/mx5, $\mathrm{mx} 7$, and $\mathrm{Ss}(\mathrm{A}) / \mathrm{SN}-\mathrm{ANS}$ measurements $(P<0.05$; Table 5$)$. An inversely related negative relation was found between facial axis $(\mathrm{BaN} / \mathrm{PtGn})$ and $\mathrm{mx} 3 \rightarrow \mathrm{mx} 7 /$ AnsPns $(P<0.05$; Table 5). A statistically significant full correlation was noted

Table 2 Mean, standard deviation ( $\pm S \mathrm{SD}$ ), and median (Me) of morphological parameters of the alveolar process of the maxilla according to Tallgren's method ${ }^{21}$ in the whole study group $(\mathrm{n}=25)$

\begin{tabular}{|c|c|c|c|}
\hline $\begin{array}{l}\text { Morphological parameters } \\
\text { characterizing the alveolar part of the } \\
\text { mandible according to Tallgren et } \mathrm{al}^{21}\end{array}$ & $\begin{array}{l}\text { Mean } \\
\text { values }\end{array}$ & $\pm \mathbf{S D}$ & $\mathrm{Me}$ \\
\hline \multicolumn{4}{|c|}{ Orientation with respect to AnsPns reference line, mm } \\
\hline $\mathrm{mxl}$ & 9.29 & 3.25 & 10.56 \\
\hline $\mathrm{m} \times 2$ & 11.72 & 3.88 & 12.13 \\
\hline $\mathrm{m} \times 3$ & 13.19 & 4.18 & $13.8 \mid$ \\
\hline $\mathrm{m} \times 4$ & 15.25 & 3.91 & 16.00 \\
\hline $\mathrm{m} \times 5$ & 15.26 & 3.85 & 15.19 \\
\hline$m \times 6$ & 15.96 & 4.13 & 15.88 \\
\hline $\mathrm{m} \times 7$ & 14.02 & 3.96 & 13.63 \\
\hline $\mathrm{Ss}(\mathrm{A})$ & 5.18 & 2.62 & 5.75 \\
\hline \multicolumn{4}{|c|}{ Orientation with respect to the SNAns reference line, $\mathrm{mm}$} \\
\hline $\mathrm{m} \times 5$ & 11.21 & 2.83 & 10.00 \\
\hline $\mathrm{m} \times 6$ & 8.79 & 2.4 & 7.75 \\
\hline $\mathrm{m} \times 7$ & 6.75 & 2.6 & 5.38 \\
\hline $\operatorname{Ss}(\mathrm{A})$ & 8.04 & 2.18 & 8.13 \\
\hline \multicolumn{4}{|l|}{ Others } \\
\hline PP-A & 15.05 & 3.11 & $|4.3|$ \\
\hline
\end{tabular}

Notes: AnsPns line, line between spina nasalis anterior and spina nasalis posterior. $\mathrm{mxl}, \mathrm{m} \times 2, \mathrm{mx} 3, \mathrm{~m} \times 4, \mathrm{~m} \times 5$, points within the contour of the alveolar process located at equal distances from each other with respect to the AnsPns line. $m \times 6$, point situated within the top of the anterior part of the alveolar ridge in relation to the AnsPns line. $m \times 7$, the most inferior point on the anterior contour of the maxillary process. Ss $(A)$, point located between anterior nasal spine and prosthion $(m \times 7)$ as the lowest point of the curve (Ans) and prosthion $(m \times 7)$ as the lowest point of the curve. PP-A, cross section of the anterior part of the alveolar process, dimension between PP and subspinale. SNAns line, line between nasion and spina nasalis anterior related to anterior cranial base (line between sella and nasion). 
Table 3 Correlations of morphological parameters of the edentulous ridge of the maxilla (according to Tallgren et $\mathrm{a}^{2 \mathrm{I}}$ ) with age, complete denture-wearing time, and the edentulous period in the whole study group ( $n=25$; table contains Spearman's rank correlation coefficient $r$ )

\begin{tabular}{|c|c|c|c|c|c|c|}
\hline \multirow{2}{*}{$\begin{array}{l}\text { Morphological parameters } \\
\text { characterizing the alveolar part of the } \\
\text { mandible according to Tallgren et } a^{21}\end{array}$} & \multicolumn{2}{|l|}{ Age } & \multicolumn{2}{|c|}{ Denture-wearing time } & \multicolumn{2}{|c|}{ Edentulous period } \\
\hline & $r$ & $P$-value & $r$ & $P$-value & $r$ & $P$-value \\
\hline \multicolumn{7}{|c|}{ Orientation with respect to AnsPns reference line } \\
\hline $\mathrm{mxl}$ & $-0.5776^{*}$ & 0.0031 & -0.3131 & 0.1363 & -0.2847 & 0.1776 \\
\hline$m \times 2$ & $-0.5302 *$ & 0.0077 & -0.2394 & 0.2599 & -0.2439 & 0.2508 \\
\hline $\mathrm{m} \times 3$ & $-0.4728^{*}$ & 0.0197 & -0.2907 & 0.1682 & -0.2705 & 0.2011 \\
\hline $\mathrm{m} \times 4$ & $-0.4055^{*}$ & 0.0493 & -0.2056 & 0.3352 & -0.3139 & 0.1353 \\
\hline $\mathrm{m} \times 5$ & -0.3322 & 0.1128 & -0.2333 & 0.2725 & -0.3597 & 0.0843 \\
\hline$m \times 6$ & -0.2321 & 0.2751 & -0.1574 & 0.4627 & -0.3450 & 0.0987 \\
\hline $\mathrm{m} \times 7$ & -0.1567 & 0.4645 & -0.0876 & 0.6841 & -0.2903 & 0.1687 \\
\hline $\mathrm{Ss}(\mathrm{A})$ & -0.1715 & 0.4230 & -0.1656 & 0.4392 & -0.1087 & 0.6132 \\
\hline \multicolumn{7}{|c|}{ Orientation with respect to SNAns reference line } \\
\hline $\mathrm{m} \times 5$ & -0.2672 & 0.2068 & -0.0991 & 0.6448 & -0.2525 & 0.2339 \\
\hline $\mathrm{m} \times 6$ & -0.1792 & 0.4021 & 0.0037 & 0.9862 & -0.1081 & 0.6150 \\
\hline $\mathrm{m} \times 7$ & -0.1828 & 0.3927 & 0.0815 & 0.7051 & -0.0295 & 0.8913 \\
\hline Ss $(A)$ & -0.2217 & 0.2979 & -0.1208 & 0.5739 & -0.1000 & 0.6421 \\
\hline \multicolumn{7}{|l|}{ Others } \\
\hline PP-A & -0.3101 & 0.1403 & -0.0950 & 0.6587 & -0.1746 & 0.4146 \\
\hline
\end{tabular}

Notes: $* P<0.05$ statistical significance. AnsPns line, line between spina nasalis anterior and spina nasalis posterior. $\mathrm{m} \times \mathrm{l}, \mathrm{m} \times 2, \mathrm{~m} \times 3, \mathrm{~m} \times 4, \mathrm{~m} \times 5$, points within the contour of the alveolar process located at equal distances from each other with respect to the AnsPns line. mx6, point situated within the top of the anterior part of the alveolar ridge in relation to the AnsPns line. $m \times 7$, the most inferior point on the anterior contour of the maxillary process. Ss(A), point located between anterior nasal spine and prosthion $(\mathrm{m} \times 7)$ as the lowest point of the curve (Ans) and prosthion $(\mathrm{m} \times 7)$ as the lowest point of the curve. PP-A, cross section of the anterior part of the alveolar process, dimension between PP and subspinale. SNAns line, line between nasion and spina nasalis anterior related to anterior cranial base (line between sella and nasion).

between $\mathrm{mx} 1 \rightarrow \mathrm{mx} 6 /$ AnsPns and $\mathrm{md} 4, \mathrm{md} 5, \mathrm{md} 6, \mathrm{md} 7$, and $\mathrm{Sm}(\mathrm{B}) / \mathrm{ML}$ parameters, which characterize the edentulous lower jaw $(P<0.05$; Table 6$)$. Measurements with respect to MLP established a close relationship between md6, $\mathrm{md} 7, \mathrm{Sm}(\mathrm{B})$, and $\mathrm{mx} 2 \rightarrow \mathrm{mx} 4 /$ AnsPns parameters.

\section{Discussion}

Comparative analysis of the initial and final AnsXiPm angles allowed an objective evaluation of correctness of the treatment, including control of the anterior total and lower facial height. Results after therapy were in good agreement with the norm proposed by Ricketts $\left(46^{\circ} \pm 3^{\circ}\right)$, noted in people with natural dentition ${ }^{16-19}$ and Brzoza et al's reference values observed in edentulous patients $\left(47^{\circ} \pm 4^{\circ}\right) .{ }^{22}$ At the time of enrollment, the mean AnsXiPm measurement was $41.68^{\circ}$; after prosthetic rehabilitation, it increased to $46.15^{\circ}$. Confirmation of the quality of clinical procedures allowed assessment of craniofacial links between anterior facial height and the edentulous maxilla.

The existence of a statistically significant relationship between upper jaw morphology (mx1 $\rightarrow$ mx6/AnsPns) and measurements associated with anterior total and lower facial height (G'Me', Sn'Me', NMe, AnsMe) emphasize the importance of prosthetic rehabilitation (Table 4).
The height of the vertical occlusal dimension is determined phylogenetically - it is not a random value. ${ }^{23}$ It is specified by three essential periods of development of the stomatognathic system and correlates with morphology of the edentulous jaw in accordance with study results (Table 4).

The gradual emergence of teeth in molar and premolar areas, by bringing the chewing surface to the occlusal plane, stimulates vertical development of the alveolar processes. At the same time, both the maxillary and mandibular bases move away from each other. It affects the prolongation of the NMe distance and larger mandibular inclination. In young people, it results in directional development of the facial skeleton. Physiologically, we can distinguish three stages of increase in the vertical dimension of occlusion. The first period follows primary dentition - includes deciduous teeth eruption. The appearance of the first molar in oral cavity is designated as the next stage. The third phase relates to the presence of the second molars fully erupted in the permanent dentition. The process of eruption does not stop when the permanent antagonistic teeth come into contact. ${ }^{24}$

The formation of the individual pattern of centric occlusion is a long-term process. It physiologically expires with the end of the growth period. After the time of relative stability, the stomatognathic system is changing gradually with time 


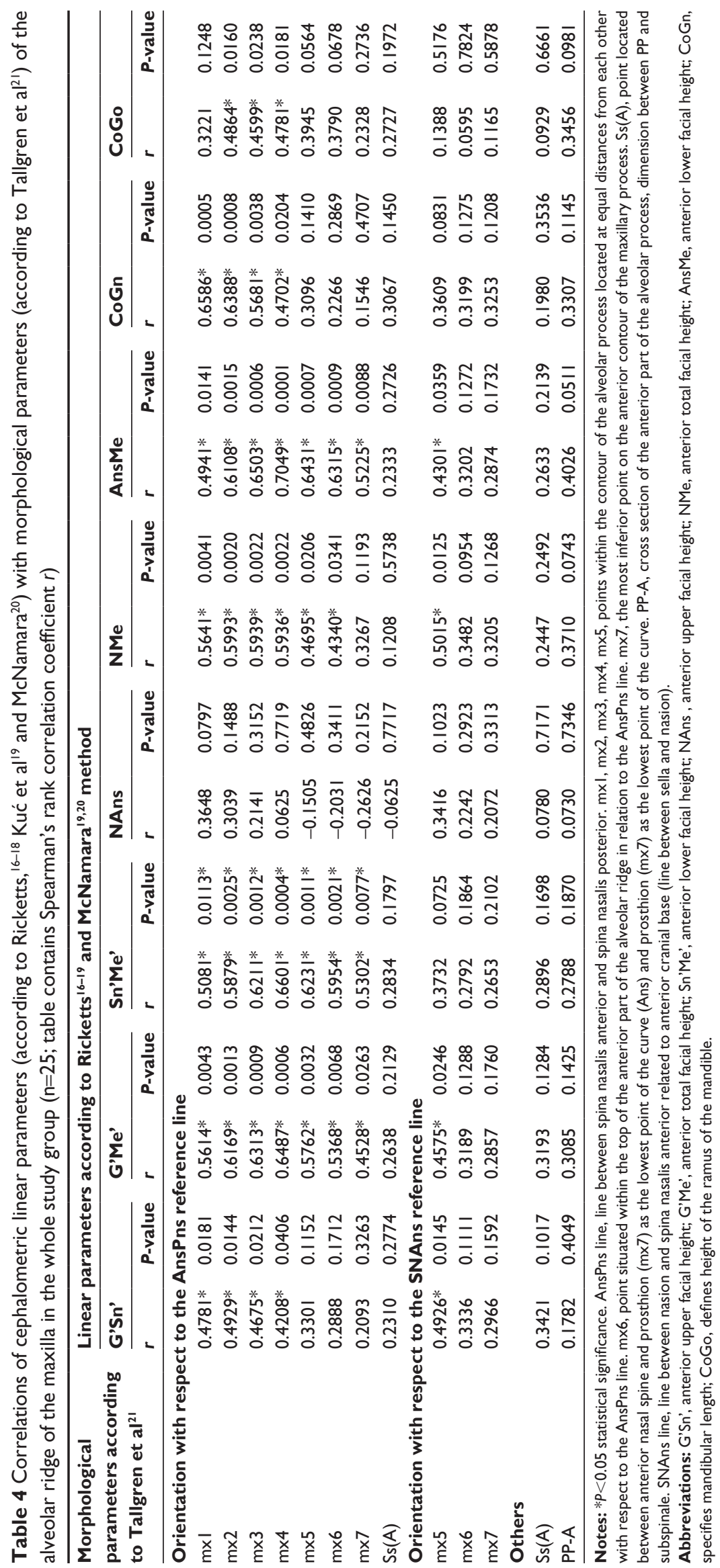




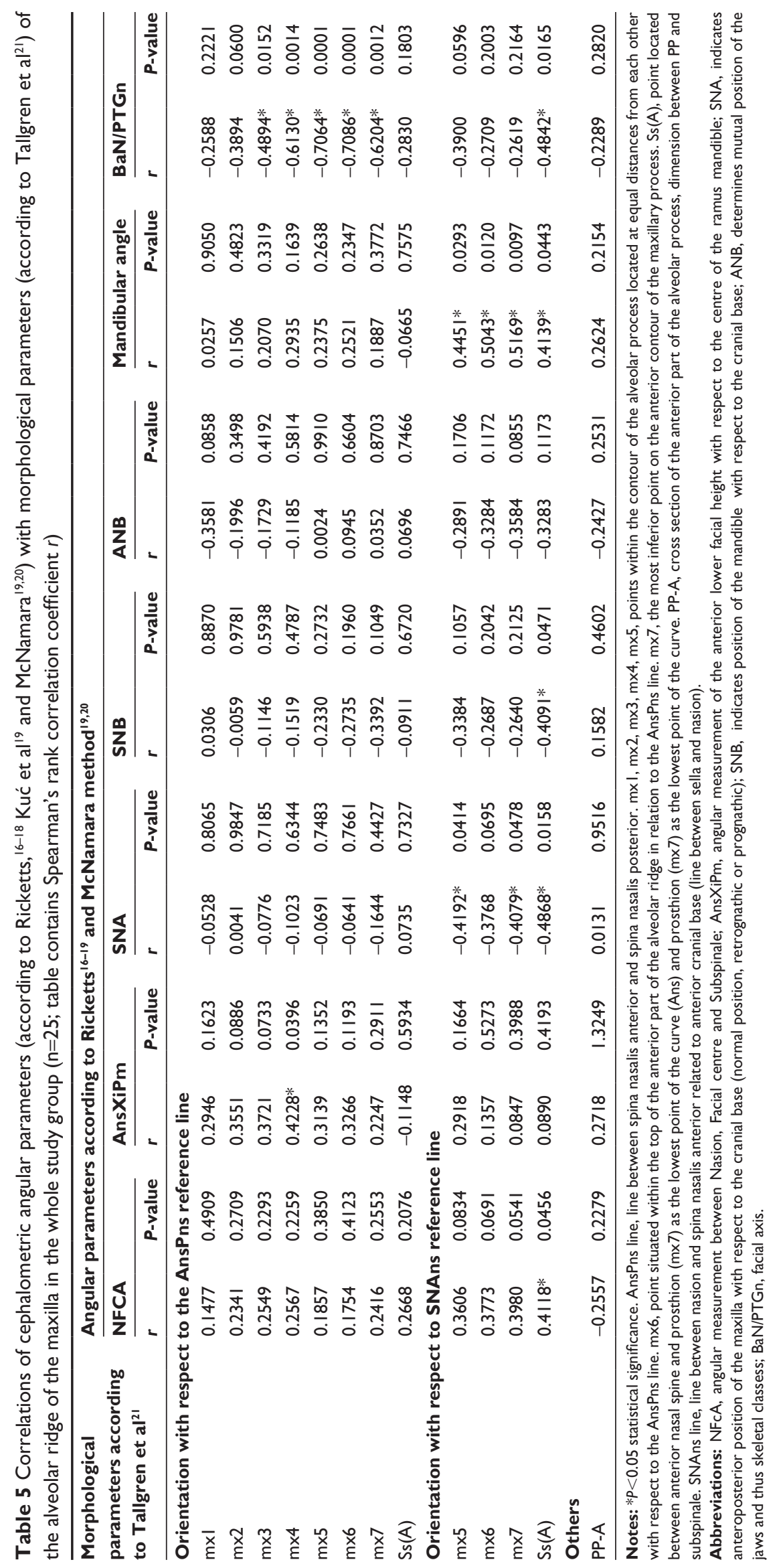




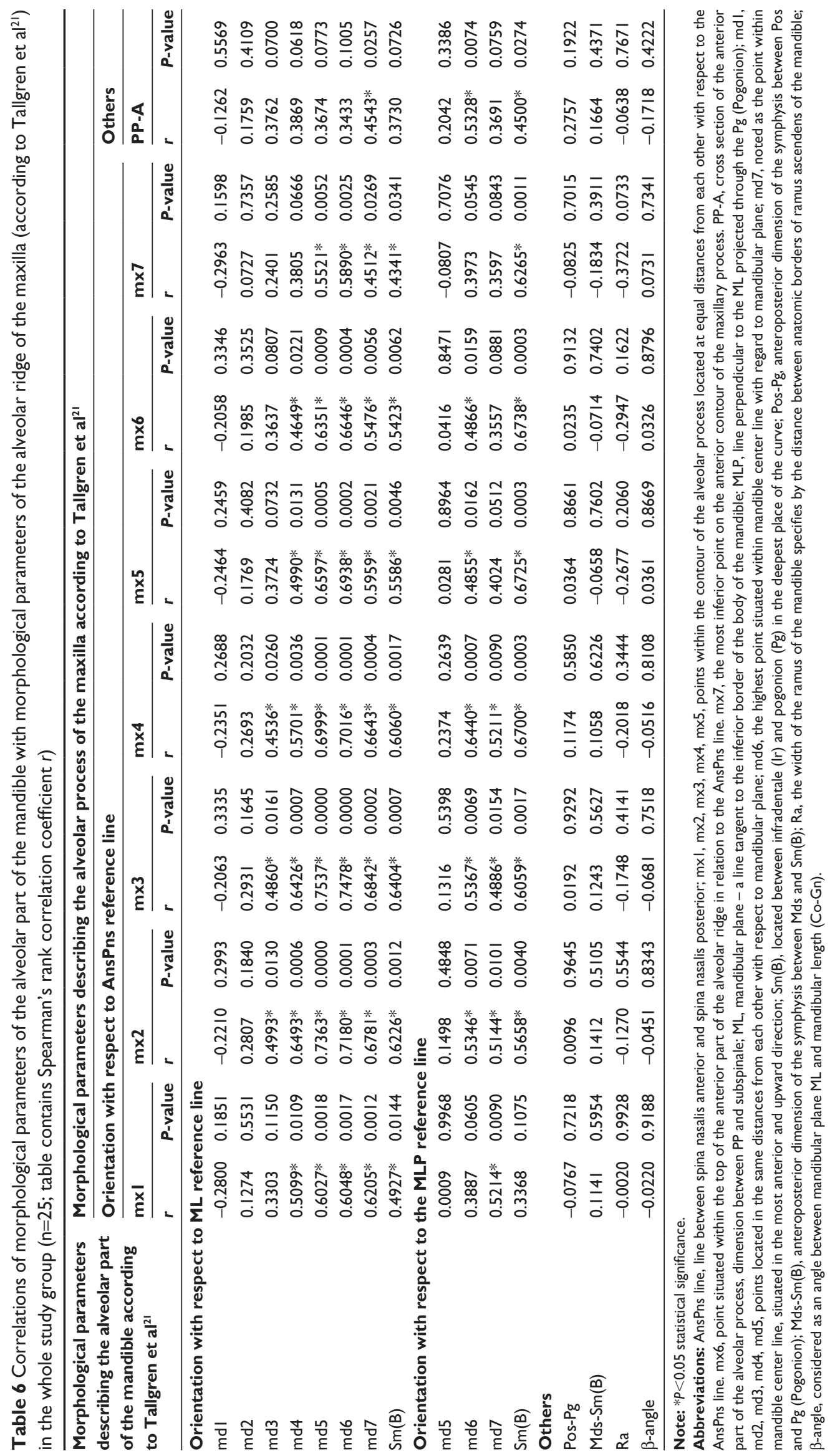


running backward or not (eg, due to tooth cusp wear) to reduce the vertical component. ${ }^{25}$

Because the distance between the nose and the chin (Sn'Me' dimension) depends on the presence of the teeth in molar and premolar areas of support, gingivo-occlusal height remains as an important factor that influences the vertical skeletal relation. Gingivo-occlusal measurement determines the course of the occlusal plane and, in turn, allows separation of the upper and lower jaw proportionally to the size of the anatomical crowns. As a result, vertical occlusal dimension of the one-third lower part of the face and, thus, the structure of the alveolar ridge of the maxilla is extended.

The length of the nasomental distance (SnMe dimension) is modified by the rotation of the growing mandible, progressing to the condyle and/or inside the matrix. In the case of posteriorotation of the mandible, the vertical component of centric occlusion increases. ${ }^{26} \mathrm{~A}$ decrease in the height is typical in the anteriorotation sample. There seems to be another mechanism that stimulates the alveolar process to elongation in the vertical direction by dismissing the maxillary and mandibular base planes. This relationship is reflected in the results obtained (Table 5). Reduction of facial axis angle (BaN/PtGn) observed in the case of dorsal rotation correlates with higher values of the parameters $\mathrm{mx} 3 \rightarrow 7 /$ AnsPns (Table 5). Increase in the length of the mandible, (CoGn $\uparrow$ ) which is often compensated by posteriorotation, remains statistically significant in comparison to the shape of posterior part of the maxilla (Table 4).

The lack of correlation between parameters $\mathrm{mx} 1 \rightarrow \mathrm{mx} 7 /$ AnsPns and the size of the angles SNA and SNB emphasizes the independence of the morphology of the alveolar process to the degree of jaw prognathion and the position of the mandible with respect to the anterior cranial fossa. On the other hand, no statistically significant implications in the ANB angle exclude the influence of skeletal class on the direction of reverse processes controlling the morphology of edentulous jaws. It should be noted that the skeletal pattern of complete denture wearers based on the measurement of ANB angle is an approximate value. The reason for this is the mobility of the mandible conditioned by involutional changes, which occur with age. The correlation in the SNA/ $\mathrm{Ss}(\mathrm{A})$ relation shows the geometric displacement of reference points in the three-dimensional space (Table 5).

Implications between the age of the patients and the vertical dimension of the alveolar bone in the distal regions $(\mathrm{mx} 1, \mathrm{mx} 2, \mathrm{mx} 3$, and $\mathrm{mx} 4)$ may indicate early molar tooth loss and could be associated with bone loss (Table 3 ). In addition, the use of partial removable prosthetic restorations in the lateral segments of the maxilla probably intensifies the rate of bone resorption as a consequence resulting from settling (Table 3).

Despite the commonly occurring morphological disproportions of the middle and lower part of the facial skeleton as described by Tallgren, ${ }^{2}$ a four times faster bone loss in the lower jaw was reported in this study, as was an interdependence of the vertical dimension of the maxilla and vertical dimension of the mandible. A full correlation was noted between all $\mathrm{mx} 1 \rightarrow \mathrm{mx} 7 / \mathrm{AnsPns}$ and $\mathrm{md} 5 \rightarrow \mathrm{md} 7$, and $\mathrm{Sm}(\mathrm{B}) / \mathrm{ML}$ parameters (Table 6). A statistically significant relationship was reported according to the anteroposterior position of indexes md6, md7, and Sm(B)/MLP and the vertical dimension of the alveolar process $\mathrm{mx} 2 \rightarrow \mathrm{mx} 4 / \mathrm{AnsPns}$ (Table 6). Considering the anatomy of the upper and lower jaw, the enormity of the factors affecting the formation of the vertical dimension in the period preceding toothlessness should be stressed. The individual profile of alveolar ridge morphology is determined largely by growth processes associated with maturation and environmental influences.

The vertical dimension of the alveolar ridge is often conditioned by the morphological abnormalities manifested by the occurrence of a deep bite with underdevelopment in the posterior segment. On the other hand, anterior or lateral open bite (with infra-occlusal space as a result of parafunction such as thumb or cheek sucking) also affects the alveolar skeletal profile..$^{27,28}$

The phenomenon of re-inclusion characterized by a progressive loss of occlusal contact contributes to reduced vertical dimension in the height of the alveolar process..$^{29,30}$ This means a gradual recession from the occlusal plane of previously partially or completely erupted teeth, with no further growth of bone in the area covered by the process. Early loss of teeth, ${ }^{31}$ bone infection, periodontal disease, ${ }^{32,33}$ residual alveolar cleft, ${ }^{34}$ post-extraction gaps, ${ }^{35}$ hypo- or oligodontia, ${ }^{36}$ and metabolic disorders, ${ }^{11}$ all disturb the natural structure of the alveolar bone and/or the growth process.

An unfavorable alveolar ridge remodeling can be observed in the case of combination syndrome (Kelly's Syndrome), ${ }^{37}$ osteoporosis, ${ }^{38-40}$ and pathological changes requiring surgical intervention associated with bone loss and phylogenesis. The latter may necessitate a change in eating habits and subsequent reduction of the length of the dental arch. Compression of the prosthetic field as a result of prosthesis settling, ${ }^{41}$ extraction (including situations requiring an immediate denture), an adaptation of the alveolus after the extraction, ${ }^{42}$ or osteonecrosis ${ }^{43}$ could also cause bone resorption. 
The following factors exert positive effects on alveolar bone elongation in the vertical direction: controlled extrusion of teeth as preparation for implanto-prosthetic treatment, ${ }^{44,45}$ extruding various segments of the dental arch during orthodontic treatment, ${ }^{46,47}$ use of bone distractors, ${ }^{48}$ regenerative procedures with bone substitute materials, ${ }^{49}$ and, in some cases, a passive eruption of teeth.

This study showed that, despite many factors that influence the morphology of the maxilla, it is possible - as in the case of the mandible ${ }^{19}$ - to find a relationship between craniofacial morphology and the structure of the alveolar ridge in edentulous complete denture wearers. Thus, these observations emphasize the uniqueness and the lack of randomness in the organization of anatomical structures with respect to involutional changes. To determine the vertical component of centric occlusion according to alveolar process morphology, further research would be required.

Cephalometric analysis of edentulous people allows monitoring of changes caused by prosthetic rehabilitation. It seems to be a prognostic factor for therapy results. Nowadays, this procedure is not included in the diagnostic standard of edentulous patients and, thus, does not prejudge the methods of treatment. There are no clearly established rules governing the kind of cephalometric measurements evaluated in scientific research in complete denture wearers.

\section{Conclusion}

The height of the lateral part of the alveolar ridge of the maxilla remains in connection with the anterior total and lower facial height obtained in the course of prosthetic rehabilitation.

The vertical dimension of the alveolar ridge of the maxilla seems to be in close relationship with the height of the anterior and lateral parts of the mandible and its body.

\section{Abbreviations}

G'Me', linear distance between the glabella and menton; Sn'Me', linear distance between subnasale and menton; NMe, linear distance between nasion and menton; AnsMe, linear distance between spina nasalis anterior and menton; AnsX$\mathrm{iPm}$, angular measurement between spina nasalis anterior, xilion, and suprapogonion; G'Sn', linear distance between glabella and subnasale; SNA, angular measurement between sella, nasion, and subspinale; NFcA, angular measurement between nasion, facial center, and subspinale; NAns, linear distance between nasion and spina nasalis anterior; $\mathrm{BaN}$, line between basion and nasion; PTGn, line between PT point and gnathion; SNB, angular measurement between sella, nasion, and supramentale; ANB, angular measurement between the anterior nasal spine, nasion, and supramentale; $\mathrm{CoGn}$, linear distance between condylion and gnathion (mandibular length); CoGo, linear distance between condylion and gonion; AnsPns line, line comprised between anterior nasal spine and posterior nasal spine; $\mathrm{Pg}$, the most anterior point on the mandible in the midline (Pogonion); ML, mandibular plane; $\mathrm{mx} 1 \rightarrow \mathrm{mx} 7$, reference points located within the alveolar process; SNAns line, line between nasion and spina nasalis anterior related to anterior cranial base (line between sella and nasion); Me, median; $\mathrm{mx} 1 \rightarrow \mathrm{mx} 6 /$ AnsPns, reference points of the alveolar process with respect to the spina nasalis anterior (Ans) and spina nasalis posterior (Pns) line; md4, reference point located within alveolar part of the mandible with respect to the mandibular plane; md5, point situated within the mandible center line with regard to the mandibular plane, noted in the most posterior and upward direction; md6, the highest point situated within the mandible center line with regard to the mandibular plane; md7, noted as the point within the mandible center line, situated in the most anterior and upward direction; MLP, line perpendicular to the mandibular plane (ML) projected through the pogonion (Pg).

\section{Acknowledgments}

This material is based on statutory work supported by the Medical University of Bialystok, Poland (no 123-08836). Any opinions, findings, and conclusions expressed in this study are those of the authors and do not necessarily reflect those of the Medical University of Bialystok, Poland.

\section{Disclosure}

The authors report no conflicts of interest in this work.

\section{References}

1. Tallgren A. Alveolar bone loss in denture wearers as related to facial morphology. Acta Odontol Scand. 1970;28(2):251-270.

2. Tallgren A. The continuing reduction of the residual alveolar ridges in complete denture wearers: a mixed-longitudinal study covering 25 years. J Prosthet Dent. 2003;89(5):427-435.

3. Tallgren A. The reduction in face height of edentulous and partially edentulous subjects during long-term denture wear. A longitudinal roentgenographic cephalometric study. Acta Odontol Scand. 1966; 24(2):195-239.

4. Tallgren A. The effect of denture wearing on facial morphology. A 7-year longitudinal study. Acta Odontol Scand. 1967;25(5):563-592.

5. Tallgren A. Positional changes of complete dentures. A 7-year longitudinal study. Acta Odontol Scand. 1969;27(5):539-561.

6. Douglass JB, Meader L, Kaplan A, Ellinger CW. Cephalometric evaluation of the changes in patients wearing complete dentures: a 20-year study. J Prosthet Dent. 1993;69(3):270-275.

7. Lang BR. Chapter 15, Occlusion for the edentulous patients. In: Zarb GA, Bolender CHL, Carlsson GE, Boucher CO, editors. Boucher's Prosthodontic Treatment for Edentulous Patients. 11th ed. St. Louis: Mosby Inc; 1997:263-278. 
8. Pietrokovski J, Starinsky R, Arensburg B, Kaffe I. Morphologic characteristics of bony edentulous jaws. J Prosthodont. 2007;16(2):141-147.

9. Cawood JI, Howell RA. A classification of the edentulous jaws. Int $J$ Oral Maxillofac Surg. 1988;17(4):232-236.

10. Canger EM, Celenk P. Radiographic evaluation of alveolar ridge heights of dentate and edentulous patients. Gerodontology. 2012;29(1):17-23.

11. Atwood DA. Some clinical factors related to rate of resorption of residual ridges. J Prosthet Dent. 1962;12(3):441-450.

12. Carlsson GE. Responses of jawbone to pressure. Gerodontology. 2004; 21(2):65-70.

13. Atwood DA. Reduction of residual ridges: a major oral disease entity. J Prosthet Dent. 1971;26(3):266-279.

14. Xie Q, Wolf J, Ainamo A. Quantitative assessment of vertical heights of maxillary and mandibular bones in panoramic radiographs of elderly dentate and edentulous subjects. Acta Odontol Scand. 1997;55(3):155-161.

15. Maruo Y, Sato T, Hara T, Shirai H. The effect of diabetes mellitus on histopathological changes in the tissues under denture base bearing masticatory pressure. J Oral Rehabil. 1999;26(4):345-355.

16. Ricketts RM. Perspectives in the clinical application of cephalometrics. Angle Orthod. 1981;51(2):115-150.

17. Ricketts RM. The role of cephalometrics in prosthetic diagnosis. $J$ Prosthet Dent. 1956;6(4):488-503.

18. Ricketts RM. The value of cephalometrics and computerized technology. Angle Orthod. 1972;42(3):179-199.

19. Kuć J, Sierpińska T, Gołębiewska M. The relationship between facial morphology and the structure of the alveolar part of the mandible in edentulous complete denture wearers. A preliminary study. Acta Odontol Scand. 2015;73(1):57-66.

20. McNamara JA Jr. A method of cephalometric evaluation. Am J Orthod. 1984;86(6):449-469.

21. Tallgren A, Lang BR, Walker GF, Ash MM Jr. Roentgen cephalometric analysis of ridge resorption and changes in jaw and occlusal relationships in immediate complete denture wearers. J Oral Rehabil. 1980;7(1):77-94.

22. Brzoza D, Barrera N, Contasti G, Hernández A. Predicting vertical dimension with cephalograms, for edentulous patients. Gerodontology. 2005;22(2):98-103.

23. Pokorny PH, Wiens JP, Litvak H. Occlusion for fixed prosthodontics: a historical perspective of the gnathological influence. J Prosthet Dent. 2008;99(4):299-313.

24. Kaifu Y, Kasai K, Townsend GC, Richards LC. Tooth wear and the "design" of the human dentition: a perspective from evolutionary medicine. Am J Phys Anthropol. 2003;122(Suppl 37):47-61.

25. Johansson A, Johansson AK, Omar R, Carlsson GE. Rehabilitation of the worn dentition. J Oral Rehabil. 2008;35(7):548-566.

26. Björk A. Prediction of mandibular growth rotation. Am J Orthod. 1969;55(6):585-599.

27. Beckmann SH, Kuitert RB, Prahl-Andersen B, Segner D, The RPS, Tuinzing DB. Alveolar and skeletal dimensions associated with overbite. Am J Orthod Dentofac Orthop. 1998;113(4):443-452.

28. Kuitert R, Beckmann S, van Loenen M, Tuinzing B, Zentner A. Dentoalveolar compensation in subjects with vertical skeletal dysplasia. Am J Orthod Dentofacial Orthop. 2006;129(5):649-657.
29. Proff P, Bayerlein T, Fanghänel J, Allegrini S Jr, Gedrange T. Morphological and clinical considerations of first and second permanent molar eruption disorders. Ann Anat. 2006;188(4):353-361.

30. Becktor KB, Bangstrup MI, Rølling S, Kjaer I. Unilateral primary or secondary retention of permanent teeth, and dental malformations. Eur J Orthod. 2002;24(2):205-214.

31. Bodic F, Hamel L, Lerouxel E, Baslé MF, Chappard D. Bone loss and teeth. Joint Bone Spine. 2005;72(3):215-221.

32. Mendieta C, Miranda J, Brunet LI, Gargallo J, Berini L. Alveolar bone necrosis and tooth exfoliation following herpes zoster infection: a review of the literature and case report. J Periodontol. 2005;76(1):148-153.

33. Cochran DL. Inflammation and bone loss in periodontal disease. J Periodontol. 2008;79(8 Suppl):1569-1576.

34. Goodacre T, Swan MC. Cleft lip and palate: current management. Paediatr Child Health. 2012;22(4):160-168.

35. Van der Weijden F, Dell'Acqua F, Slot DE. Alveolar bone dimensional changes of post-extraction sockets in humans: a systematic review. $J$ Clin Periodontol. 2009;36(12):1048-1058.

36. Worsaae N, Jensen BN, Holm B, Holsko J. Treatment of severe hypodontia-oligodontia - an interdisciplinary concept. Int $J$ Oral Maxillofac Surg. 2007;36(6):473-480.

37. Palmqvist S, Carlsson GE, Owall B. The combination syndrome: a literature review. J Prosthet Dent. 2003;90(3):270-275.

38. Dervis E. Oral implications of osteoporosis. Oral Surg Oral Med Oral Pathol Oral Radiol Endod. 2005;100(3):349-356.

39. Jeffcoat M. The association between osteoporosis and oral bone loss. J Periodontol. 2005;76(11 Suppl):2125-2132.

40. Kaye EK. Bone health and oral health. J Am Dent Assoc. 2007;138(5): 616-619.

41. Wyatt CC. The effect of prosthodontic treatment on alveolar bone loss: a review of the literature. J Prosthet Dent. 1998;80(3):362-366.

42. Schropp L, Wenzel A, Kostopoulos L, Karring T. Bone healing and soft tissue contour changes following single-tooth extraction: a clinical and radiographic 12-month prospective study. Int J Periodontics Restorative Dent. 2003;23(4):313-323.

43. Migliorati CA, Siegel MA, Elting LS. Bisphosphonate-associated osteonecrosis: a long-term complication of bisphosphonate treatment. Lancet Oncol. 2006;7(6):508-514.

44. Chambrone L, Chambrone LA. Forced orthodontic eruption of fractured teeth before implant placement: case report. J Can Dent Assoc. 2005;71(4):257-261.

45. Brindis MA, Block MS. Orthodontic tooth extrusion to enhance soft tissue implant esthetics. J Oral Maxillofac Surg. 2009;67(11 Suppl): 49-59.

46. Bach N, Baylard JF, Voyer R. Orthodontic extrusion: periodontal considerations and applications. J Can Dent Assoc. 2004;70(11):775-780.

47. Isaacson RJ, Lindauer SJ. Closing anterior open bites: the extrusion arch. Semin Orthod. 2001;7(1):34-41.

48. Liou EJW, Chen PKT. Intraoral distraction of segmental osteotomies and miniscrews in management of alveolar cleft. Semin Orthod. 2009; 15(4):257-267.

49. Zitzmann NU, Schärer $P$, Marinello $C P$, Schüpbach $P$, Berglundh T. Alveolar ridge augmentation with Bio-Oss: a histologic study in humans. Int J Periodontics Restorative Dent. 2001;21(3):288-295.
Clinical Interventions in Aging

\section{Publish your work in this journal}

Clinical Interventions in Aging is an international, peer-reviewed journal focusing on evidence-based reports on the value or lack thereof of treatments intended to prevent or delay the onset of maladaptive correlates of aging in human beings. This journal is indexed on PubMed Central, MedLine,

\section{Dovepress}

CAS, Scopus and the Elsevier Bibliographic databases. The manuscript management system is completely online and includes a very quick and fair peer-review system, which is all easy to use. Visit http://www.dovepress. com/testimonials.php to read real quotes from published authors. 